\title{
The Global Dynamics of HIV Latency Model Including Cell-to-Cell Viral Transmission
}

\author{
Wajahat Ali", Zhipeng Qiu \\ School of Science, Nanjing University of Science and Technology, Nanjing, China
}

Email address:

wajahatali.kh@gmail.com (W. Ali)

${ }^{*}$ Corresponding author

\section{To cite this article:}

Wajahat Ali, Zhipeng Qiu. The Global Dynamics of HIV Latency Model Including Cell-to-Cell Viral Transmission. Applied and Computational Mathematics. Vol. 10, No. 4, 2021, pp. 91-99. doi: 10.11648/j.acm.20211004.12

Received: May 29, 2021; Accepted: June 21, 2021; Published: July 9, 2021

\begin{abstract}
HIV spreads by cell-to-cell transfer and the release of cell-free particles. A slightly more effective method of retroviral transmission is the direct cell-to-cell transfer of HIV, according to recent reports. Intracellular interaction between unhealthy and healthy cells, in combination with cytokine discharged by the cells included, may affect the susceptibility of a target resting CD4+T cell to HIV infection and the formation of latent infection. We suggest a class of HIV latency mathematical model, integrating both cell-free virus transmission and direct cell-to-cell diffusion to improve the understanding of the dynamics of the latent reservoirs. We incorporate four components in our model: the uninfected $\mathrm{T}$ cells, the latently infected $\mathrm{T}$ cells, the active-infected $\mathrm{T}$ cells and the HIV viruses. We examine the latency model by introducing the basic reproduction number. We first establish the non-negativity and boundedness of the solutions of the system, and then we investigate the global stability of the steady states. The diseased-free equilibrium is globally stable when the basic reproduction number is less than 1 and if the basic reproduction number is greater than 1, the diseased equilibrium exists and is globally stable. Numerical simulations are executed to interpret the theoretical outcomes and evaluate the relative contribution of latency fractions in the virus production and the HIV latent reservoir by providing estimates.
\end{abstract}

Keywords: HIV, Infected Latent Cells, Virus Intracellular Transmission, Global Stability

\section{Introduction}

The manner in which a pathogen evolves inside a host has significant implications for the effective establishment of an infection and its maintenance. Viruses have developed distinct mechanisms to attack the target cells so as for replication and infection dissemination.

In general, two main ways are identified for viral transmission, i.e. (i) virus cell-free (CF) transmission and (ii) virus cell-cell (CC) transmission [3]. Although $\mathrm{CF}$ viral particle diffusion allows distant cell infection whereas $\mathrm{CC}$ viral transmission relates to local diffusion, ignores complex operations of our body and is considered to have the ability to guard the viral particles from neutralization of antibodies, antiviral constraint aspects and few anti-retroviral remedies $[4,5]$. Therefore, the CC transmission way is thought to be more responsible in spreading infection $[6,7]$.

In addition, virally induced bonds, or virological synapses are formed by cell contacts, among CD4+T cells, concentrating immense amount of particles at the place of intracellular contact [8-10]. The target cells receive a high MOI due to this process [2, 11]. It is still necessary to determine in detail the importance and input of every transmission modes to viral spread. However, a decent quantification of the transmission dynamics is required to address it.

Computational models formulated to observe the infection kinetics of HIV, containing both these transmission ways [1, 14-18, 31-41, 43, 46]. The relative contribution to the spread of HIV by the two transmission modes is studied by Komarova et al. [13] and Iwami et al. [12]. Lai [20, 21] derived models including both these ways of viral diffusion. H. Pourbashash et al. [17] analyzed a multi-strain model to show a competitive exclusion principle.

The highly suggestive obstacle for the HIV-1 infection wipeout is the presence of latent infected pools which subsist regardless of long-term viral multiplication restraint by HAART [22-24]. Most of infected latent cells are composed 
of long living resting CD4+T cells and are managed by homeostatic proliferation [25-27]. These latent cells can produce virus infection by connecting with relevant antigens.

Motivated by the work of Alshorman et al. [28] and Lai et al. [20, 21], in this paper, we derive an HIV latency model which includes $\mathrm{CF}$ and $\mathrm{CC}$. Firstly, we prove the nonnegativity and boundedness of solutions of our model. Then, we perform the derivation of basic reproduction number $\mathcal{R}_{0}$ and equilibria. We investigate the global stability of two steady states. In the end, numerical simulations are performed to show the complexity of dynamics of model.

\section{The Model}

We developed a following mathematical model.

$$
\begin{gathered}
\frac{d T(t)}{d t}=\lambda-d_{T} T-k_{1} V T-k_{2} T T^{*}, \\
\frac{d L(t)}{d t}=\alpha_{1} k_{1} V T+\alpha_{2} k_{2} T T^{*}-d_{L} L-b L,
\end{gathered}
$$

$$
\begin{gathered}
\frac{d T^{*}(t)}{d t}=\left(1-\alpha_{1}\right) k_{1} V T+\left(1-\alpha_{2}\right) k_{2} T T^{*}-\delta T^{*}+b L \\
\frac{d V(t)}{d t}=N \delta T^{*}-c V
\end{gathered}
$$

where the concentration of latent infected cells denoted by L. The concentration of uninfected cells, infected cells and free viruses is represented by $\mathrm{T}, T^{*}$ and $\mathrm{V}$, respectively. The suspected cells become infected due to virus-to-cell and cellassociated infections at the rates $k_{1} V T$ and $k_{2} T T^{*}$, respectively, where $k_{1}$ and $k_{2}$ denotes the incidence rates. The fractions $\left(1-\alpha_{1}, 1-\alpha_{2}\right.$ and $\left.\alpha_{1}, \alpha_{2}\right)$ represents the possibilities that upon infection, a suspected cell turns out to be either productively infected or latently infected, respectively. The death rate of latently infected cells and productively infected cells is denoted by $d_{L}$ and $\delta$. Parameter $b$ represents the rate at which latently infected cells become productively infected cells. We did not add the proliferation of latently infected cells in this model but can do so as in [42].

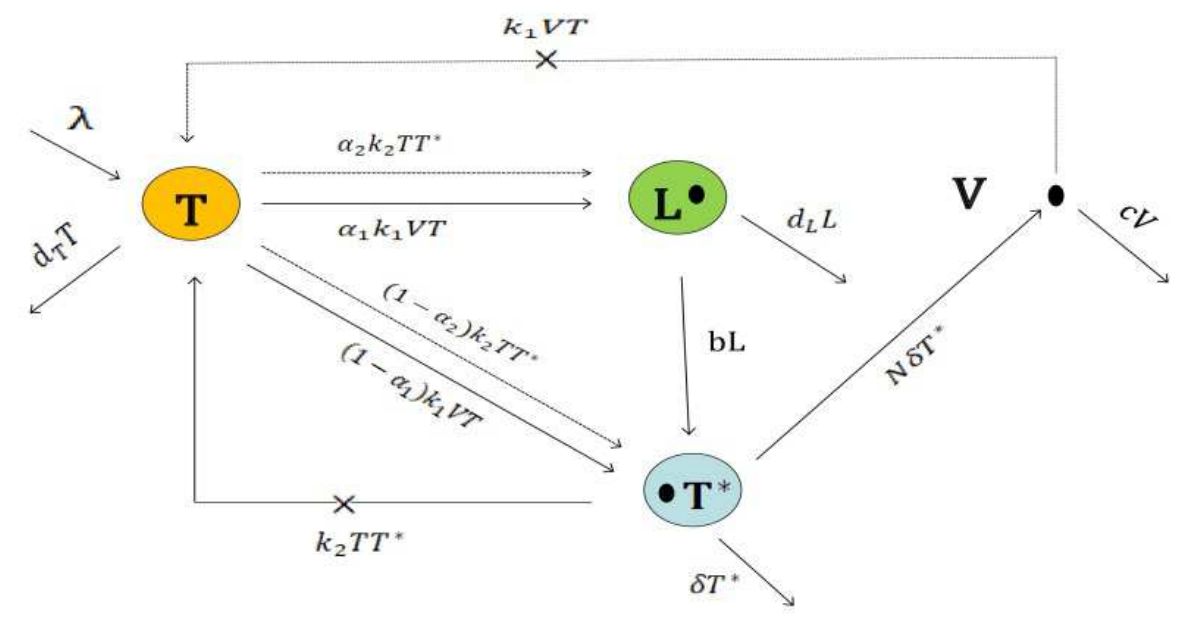

Figure 1. Model (1) illustrative representation. Variables $T, L, T^{*}$ and $V$ represent infection-free $C D 4+T$ cells, infected CD4+ T latent cells, productively unhealthy $C D 4+T$ cells and $C F$, respectively.

\subsection{Non-negativity and Boundedness}

Let us define

\begin{tabular}{|c|c|c|c|c|}
\hline Parameter & Description & Rate & Units & Fountains \\
\hline$k_{1}$ & T-cells Infection proportion for CF & $10^{-10} \sim 10^{-8}$ & $d^{-1} m l$ & {$[48]$} \\
\hline$k_{2}$ & $\mathrm{~T}$-cells Infection proportion for $\mathrm{CC}$ & $10^{-8} \sim 10^{-6}$ & $d^{-1} m l$ & {$[17]$} \\
\hline$\lambda$ & $\mathrm{T}$-cells origination proportion & $10^{4}$ & $d^{-1} m l^{-1}$ & [47] \\
\hline$b$ & T-cells (latent) stimulation proportion & 0.01 & $d^{-1}$ & {$[42]$} \\
\hline$d_{L}$ & Infected T-cells (latent) mortality proportion & 0.004 & $d^{-1}$ & [19] \\
\hline$\delta$ & T-cells (infected) mortality proportion & 1 & $d^{-1}$ & [36] \\
\hline$\alpha_{2}$ & Latency proportion for $\mathrm{CC}$ & 0.001 & No unit & [49] \\
\hline$N$ & Viral explode proportion & $100 \sim 2000$ & $d^{-1}$ cell $^{-1}$ & {$[42,47]$} \\
\hline$c$ & Virions mortality proportion & 23 & $d^{-1}$ & [36] \\
\hline
\end{tabular}

$$
\Omega=\left\{\left(T, L, T^{*}, V\right) \in \mathbb{R}_{\geq 0}^{4}: 0 \leq T, L, T^{*} \leq M_{1}, 0 \leq V \leq M_{2}\right\} .
$$

Lemma 2.1. The compact set $\Omega$ is positively invariant for system (1).

Table 1. System framework. 
Proof.

We observe that

$$
\begin{gathered}
\left.\dot{T}\right|_{(T=0)}=\lambda>0, \\
\left.\dot{L}\right|_{(L=0)}=\left(\alpha_{1} k_{1} V+\alpha_{2} k_{2} T^{*}\right) T \geq 0, \forall T, T^{*}, V \geq 0, \\
\left.T^{*}\right|_{\left(T^{*}=0\right)}=\left(1-\alpha_{1}\right) k_{1} V T+b L, \forall T, L, V \geq 0, \\
\left.\dot{T}\right|_{(V=0)}=N \delta T^{*}, \forall T^{*} \geq 0 .
\end{gathered}
$$

This confirms that $\left(T(t), L(t), T^{*}(t), V(t)\right) \in \mathbb{R}_{\geq 0}^{4}$ with $\left(T(0), L(0), T^{*}(0), V(0)\right) \in \mathbb{R}_{\geq 0}^{4}$. Let $G=T+L+T^{*}+\frac{1}{2 N} V$. Then

$$
\begin{gathered}
\dot{G}=\lambda-d_{T} T-k_{1} V T-k_{2} T T^{*}+\alpha_{1} k_{1} V T+\alpha_{2} k_{2} T T^{*}-d_{L} L-b L \\
+\left(1-\alpha_{1}\right) k_{1} V T+\left(1-\alpha_{2}\right) k_{2} T T^{*}-\delta T^{*}+b L+\frac{1}{2 N}\left(N \delta T^{*}-c V\right) \\
=\lambda-d_{T} T-d_{L} L-\delta T^{*}-\frac{c V}{2 N} \\
\leq \lambda-\sigma\left(T+L+T^{*}-\frac{1}{2 N} V\right)=\lambda-\sigma G,
\end{gathered}
$$

where $\sigma=\min \left\{d_{T}, d_{L}, \delta, c\right\}$. Hence $0 \leq G(t) \leq M_{1}$ for all $t \geq 0$ if $G(0) \leq M_{1}$, where $M_{1}=\frac{\lambda}{\sigma}$. Consequently, $0 \leq T, L, T^{*} \leq$ $M_{1}$ and $0 \leq V \leq M_{2} \forall t \geq 0$ if $T(0)+L(0)+T^{*}(0)+\frac{1}{2 N} V(0) \leq M_{1}$, where $M_{2}=\frac{2 N \lambda}{\sigma}$. This establishes the boundedness of $T(t), L(t), T^{*}(t)$ and $V(t)$.

\subsection{Reproduction Numbers and Equilibria}

The equilibria of model (1) should satisfy the following equalities.

$$
\begin{gathered}
\lambda-d_{T} T-k_{1} V T-k_{2} T T^{*}=0, \\
\alpha_{1} k_{1} V T+\alpha_{2} k_{2} T T^{*}-d_{L} L-b L=0, \\
\left(1-\alpha_{1}\right) k_{1} V T+\left(1-\alpha_{2}\right) k_{2} T T^{*}-\delta T^{*}+b L=0, \\
N \delta T^{*}-c V=0 .
\end{gathered}
$$

Straightforward calculation shows that our system (1) has two following steady states.

i. the healthy equilibrium: $E_{0}=\left(T_{0}, 0,0,0\right)$, where $T_{0}=\frac{\lambda}{d_{T}}$.

ii. the chronic equilibrium: $\bar{E}=\left(\bar{T}, \bar{L}, \bar{T}^{*}, \bar{V}\right)$.

Inspired by the method in Diekmann et al. [29] and van den Driessche and Watmough [30], let us introduce the basic reproduction number for our system (1).

$$
\mathcal{R}_{0}=\frac{N k_{1} \lambda}{c d_{T}}\left(\frac{b \alpha_{1}}{b+d_{L}}+\left(1-\alpha_{1}\right)\right)+\frac{k_{2} \lambda}{\delta d_{T}}\left(\frac{b \alpha_{2}}{b+d_{L}}+\left(1-\alpha_{2}\right)\right),
$$

where $\frac{N k_{1} \lambda}{c d_{T}}$ and $\frac{k_{2} \lambda}{\delta d_{T}}$ are the basic reproduction numbers through virus-to-cell infection and cell-associated infection, respectively. We also observe that $\mathcal{R}_{0}$ determined here is similar to that attained from the threshold condition ensuring the existence of the endemic steady state.

When $\mathcal{R}_{0}>1$ then

$$
\begin{gathered}
\bar{T}=\frac{\lambda}{d_{T} \mathcal{R}_{0}}, \\
\bar{L}=\frac{\lambda\left(N \delta \alpha_{1} k_{1}+c \alpha_{2} k_{2}\right)\left(\mathcal{R}_{0}-1\right)}{\mathcal{R}_{0}\left(b+d_{L}\right)\left(N \delta k_{1}+c k_{2}\right)},
\end{gathered}
$$

$$
\begin{gathered}
\bar{T}^{*}=\frac{c d_{T}\left(\mathcal{R}_{0}-1\right)}{\left(N \delta k_{1}+c k_{2}\right)}, \\
\bar{V}=\frac{N \delta d_{T}\left(\mathcal{R}_{0}-1\right)}{\left(N \delta k_{1}+c k_{2}\right)},
\end{gathered}
$$

Theorem 2.2. We have $\mathcal{R}_{0}$ from our system (1), then

i. System (1) has just non-disease equilibrium $E_{0}$ if $\mathcal{R}_{0}<1$ :

ii. System (1) has two equilibria $E_{0}$ and $\bar{E}$ if $\mathcal{R}_{0}>1$ :

Its proof can be evaluated by simple arithmetic and we skipped it here. 


\section{Global Stability Analysis of System with $a_{1}=a_{2}$}

We formulate Lyapunov functionals to examine the global kinetics of system's (1) steady states in this section. We assume $\alpha_{1}=\alpha_{2}$ while the case of $\alpha_{1} \neq \alpha_{2}$ remains to be further studied.

Theorem 3.1. If $\mathcal{R}_{0}<1$, then the healthy equilibrium $E_{0}$ is globally asymptotically stable, while $\alpha_{1}=\alpha_{2}$.

Proof. Initially, we claim that $T(t) \leq T_{0}$ for any $t \geq 0$. Otherwise there exists $t_{1}>0$ such that $T\left(t_{1}\right)>T_{0}$ and

$$
\begin{aligned}
\frac{d T}{d t}\left(t_{1}\right) & >0 . \\
\frac{d T}{d t}\left(t_{1}\right) & =\lambda-d_{T} T\left(t_{1}\right)-k_{1} V\left(t_{1}\right) T\left(t_{1}\right)-k_{2} T\left(t_{1}\right) T^{*}\left(t_{1}\right) \leq 0,
\end{aligned}
$$

which contradicts with $\frac{d T}{d t}\left(t_{1}\right)>0$. Now, we construct the Lyapunov function

$$
X(t)=b L+\left(d_{L}+b\right) T^{*}+\frac{k_{1}\left(b+d_{L}\left(1-\alpha_{1}\right)\right) T_{0} V}{c} .
$$

Now compute the derivation of $X(t)$ along the system's (1) solution, then

$$
\begin{gathered}
\frac{d X}{d t}=b\left(\alpha_{1} k_{1} V T+\alpha_{2} k_{2} T T^{*}-d_{L} L-b L\right)+\left(d_{L}+b\right)\left(\left(1-\alpha_{1}\right) k_{1} V T\right. \\
\left.+\left(1-\alpha_{2}\right) k_{2} T T^{*}-\delta T^{*}+b L\right)+\frac{k_{1}\left(b+d_{L}\left(1-\alpha_{1}\right)\right) T_{0}}{c}\left(N \delta T^{*}-c V\right) \\
=\left(b+d_{L}\left(1-\alpha_{1}\right)\right)\left(k_{1} V T+k_{2} T T^{*}\right)-\left(b+d_{L}\right) \delta T^{*} \\
+\frac{k_{1}\left(b+d_{L}\left(1-\alpha_{1}\right)\right) T_{0}}{c}\left(N \delta T^{*}-c V\right) \\
=\left(b+d_{L}\left(1-\alpha_{1}\right)\right)\left(k_{1} V T+k_{2} T T^{*}\right)\left(T-T_{0}\right)+\left(d_{L}+b\right) \delta T^{*}\left(\mathcal{R}_{0}-1\right) .
\end{gathered}
$$

Hence, $\frac{d X}{d t} \leq 0$ when $\mathcal{R}_{0}<1$. Moreover $\frac{d X}{d t}=0$ iff $T=T_{0} ; L=0 ; T^{*}=0$ and $V=0$. Largest consistent set within $\left(T ; L ; T^{*} ; V\right): \frac{d X}{d t}=0$ be the singleton set $E_{0}$. By using LaSalle's invariance principle, we attain that the infection-free equilibrium $E_{0}$ is globally asymptotically stable [44].

Theorem 3.2. If $\mathcal{R}_{0}>1$, then the acute-infection equilibrium $\bar{E}$ is globally asymptotically stable, while $\alpha_{1}=\alpha_{2}$.

Proof. Let us formulate Lyapunov functional

$$
\begin{aligned}
Y(t) & =\left(b+d_{L}\left(1-\alpha_{1}\right)\right)\left[T(t)-\bar{T}-\bar{T} \ln \frac{T}{\bar{T}}\right]+b\left[L(t)-\bar{L}-\bar{L} \ln \frac{L}{\bar{L}}\right] \\
& +\left(b+d_{L}\right)\left[T^{*}(t)-\bar{T}^{*}-\bar{T}^{*} \ln \frac{T^{*}}{\bar{T}^{*}}\right]+\frac{k_{1}\left(b+d_{L}\left(1-\alpha_{1}\right)\right) \bar{T}}{c}\left[V(t)-\bar{V}-\bar{V} \ln \frac{V}{\bar{V}}\right]
\end{aligned}
$$

Then the derivative of Y along system's (1) solution is

$$
\begin{gathered}
\frac{d Y}{d t}=\left(b+d_{L}\left(1-\alpha_{1}\right)\right)\left(1-\frac{\bar{T}}{T}\right)\left(\lambda-d_{T} T-k_{1} V T-k_{2} T T^{*}\right)+b\left(1-\frac{\bar{L}}{L}\right)\left(\alpha_{1} k_{1} V T+\alpha_{2} k_{2} T T^{*}\right. \\
\left.-d_{L} L-b L\right)+\left(b+d_{L}\right)\left(1-\frac{\bar{T}^{*}}{T^{*}}\right)\left(\left(1-\alpha_{1}\right) k_{1} V T+\left(1-\alpha_{2}\right) k_{2} T T^{*}-\delta T^{*}+b L\right)+\frac{k_{1}\left(b+d_{L}\left(1-\alpha_{1}\right)\right) \bar{T}}{c}\left(N \delta T^{*}-c V\right)\left(1-\frac{\bar{V}}{V}\right) \\
=\left(b+d_{L}\left(1-\alpha_{1}\right)\right)\left(1-\frac{\bar{T}}{T}\right)\left(\lambda-d_{T} T-\lambda+d_{T} T\right)-k_{1}\left(b+d_{L}\left(1-a_{1}\right)\right) V T \\
-k_{2}\left(b+d_{L}\left(1-a_{1}\right)\right) T T^{*}+k_{1}\left(b+d_{L}\left(1-a_{1}\right)\right) \bar{V} \bar{T}+k_{2}\left(b+d_{L}\left(1-a_{1}\right)\right) \bar{T} \bar{T}^{*} \\
-k_{1}\left(b+d_{L}\left(1-a_{1}\right)\right) \frac{\bar{V} \bar{T}^{2}}{T}-k_{2}\left(b+d_{L}\left(1-a_{1}\right)\right) \frac{\bar{T}^{2} \bar{T}^{*}}{T}+k_{1}\left(b+d_{L}\left(1-a_{1}\right)\right) V \bar{T} \\
+k_{2}\left(b+d_{L}\left(1-a_{1}\right)\right) \bar{T} T^{*}+k_{1} \alpha_{1} b V T+k_{2} \alpha_{1} b T T^{*}-b\left(b+d_{L}\right) L+b\left(b+d_{L}\right) \bar{L} \\
-k_{1} \alpha_{1} b \frac{V T \bar{L}}{L}-k_{2} \alpha_{1} b \frac{T T^{*} \bar{L}}{L}+k_{1}\left(b+d_{L}\right)\left(1-a_{1}\right) V T+k_{2}\left(b+d_{L}\right)\left(1-a_{1}\right) T T^{*} \\
+b\left(b+d_{L}\right) L-\delta\left(b+d_{L}\right) T^{*}+\delta\left(b+d_{L}\right) \bar{T}^{*}-k_{1}\left(b+d_{L}\right)\left(1-a_{1}\right) \frac{V T \bar{T}^{*}}{T^{*}}-k_{2}\left(b+d_{L}\right) \\
\left(1-a_{1}\right) T \bar{T}^{*}-b\left(b+d_{L}\right) \frac{L \bar{T}^{*}}{T^{*}}+\frac{k_{1} N \delta\left(b+d_{L}\left(1-a_{1}\right)\right) T^{*} \bar{T}}{c}-k_{1}\left(b+d_{L}\left(1-a_{1}\right)\right) V \bar{T}
\end{gathered}
$$




$$
\begin{gathered}
+k_{1}\left(b+d_{L}\left(1-a_{1}\right)\right) \bar{V} \bar{T}-\frac{k_{1} N \delta\left(b+d_{L}\left(1-a_{1}\right) \bar{V} T^{*} \bar{T}\right.}{c V} \\
=-\left(b+d_{L}\left(1-\alpha_{1}\right)\right) d_{T}(T-\bar{T})^{2}+k_{1}\left(b+d_{L}\right)\left(1-a_{1}\right) \bar{V} \bar{T}+k_{2}\left(b+d_{L}\right)\left(1-a_{1}\right) \bar{T} \bar{T}^{*}+ \\
k_{1} \alpha_{1} b \bar{V} \bar{T}+k_{2} \alpha_{1} b \bar{T} \bar{T}^{*}-k_{1}\left(b+d_{L}\right)\left(1-a_{1}\right) \frac{\bar{V} \bar{T}^{2}}{T}-k_{1} \alpha_{1} b \frac{\bar{V} \bar{T}^{2}}{T}-k_{2}\left(b+d_{L}\right) \\
\left(1-a_{1}\right) \frac{\bar{T}^{2} \bar{T}^{*}}{T}-k_{2} \alpha_{1} b \frac{\bar{T}^{2} \bar{T}^{*}}{T}-k_{1}\left(b+d_{L}\right)\left(1-a_{1}\right) V T-k_{1} \alpha_{1} b V T-k_{2}\left(b+d_{L}\right) \\
\quad\left(1-a_{1}\right) T-k_{2} \alpha_{1} b T T^{*}+k_{1}\left(b+d_{L} V \bar{T}+k_{2}\left(b+d_{L}\left(1-\alpha_{1}\right)\right) \bar{T} T^{*}+k_{1} \alpha_{1} b V T\right. \\
+k_{2} \alpha_{1} b T T^{*}-b\left(b+d_{L}\right) L-k_{1} \alpha_{1} b \frac{V T \bar{L}}{L}-k_{2} \alpha_{1} b \frac{T T^{*} \bar{L}}{L}+k_{1} \alpha_{1} b \bar{V} \bar{T}+k_{2} \alpha_{1} b \bar{T} \bar{T}^{*} \\
+k_{1}\left(b+d_{L}\right)\left(1-a_{1}\right) V T+k_{2}\left(b+d_{L}\right)\left(1-a_{1}\right) T T^{*}+b\left(b+d_{L}\right) L-\delta\left(b+d_{L}\right) T^{*} \\
-k_{1}\left(b+d_{L}\right)\left(1-a_{1}\right) \frac{V T \bar{T}^{*}}{T^{*}}-k_{2}\left(b+d_{L}\right)\left(1-a_{1}\right) T \bar{T}^{*}+k_{1}\left(b+d_{L}\right)\left(1-a_{1}\right) \bar{V} \bar{T} \\
-k_{1} \alpha_{1} b \frac{\bar{V} \bar{T} L \bar{T}^{*}}{T^{*} \bar{L}}-k_{2} \alpha_{1} b \frac{\bar{T}_{L} \bar{T}^{* 2}}{T^{*} \bar{L}}+k_{1} \alpha_{1} b \bar{V} \bar{T}+k_{2} \alpha_{1} b \bar{T} \bar{T}^{*}+k_{2}\left(b+d_{L}\right)\left(1-a_{1}\right) \bar{T} \bar{T}^{*} \\
+\delta\left(b+d_{L}\right) T^{*}-k_{1}\left(b+d_{L}\left(1-\alpha_{1}\right)\right) \frac{\bar{V}^{2} \bar{T} T^{*}}{\bar{T}^{*} V}+k_{1}\left(b+d_{L}\right)\left(1-a_{1}\right) \bar{V} \bar{T}+k_{1} b \alpha_{1} \bar{V} \bar{T}-k_{1}\left(b+d_{L}\left(1-\alpha_{1}\right)\right) \bar{T} T^{*},
\end{gathered}
$$

where we used

$$
\left.\frac{k_{1} N \delta\left(b+d_{L}\left(1-\alpha_{1}\right)\right) T^{*} \bar{T}}{c}=\delta\left(b+d_{L}\right)-k_{2}\left(b+d_{L}\right)\left(1-\alpha_{1}\right)\right) \bar{T} T^{*},
$$

which we get by combining the following identities

$$
\begin{gathered}
\delta \bar{T}^{*}+d_{L} \bar{L}=k_{1} \bar{V} \bar{T}-k_{2} \bar{T} \bar{T}^{*}, \\
\bar{L}=\frac{\alpha_{1} \delta \bar{T}^{*}}{b+d_{L}\left(1-\alpha_{1}\right)^{\prime}} \\
\bar{T}^{*}=\frac{k_{1}\left(b+d_{L}\left(1-\alpha_{1}\right)\right) \bar{V} \bar{T}}{\delta\left(b+d_{L}\right)-k_{2}\left(b+d_{L}\left(1-\alpha_{1}\right)\right) \bar{T}}, \\
N \delta \bar{T}^{*}=c \bar{V} .
\end{gathered}
$$

Thus equation becomes

$$
\begin{gathered}
\left.\frac{d Y}{d t}=-\left(b+d_{L}\left(1-\alpha_{1}\right)\right) d_{T}(T-\bar{T})^{2}+k_{1}\left(b+d_{L}\right)\left(1-\alpha_{1}\right)\right) \bar{V} \bar{T}\left(3-\frac{\bar{T}}{T}-\frac{T V \bar{T}^{*}}{\bar{V} \bar{T} T^{*}}-\frac{\bar{V} T^{*}}{V \bar{T}^{*}}\right) \\
\left.+k_{1} b \alpha_{1} \frac{\bar{V}}{\bar{T}}\left(4-\frac{\bar{T}}{T}-\frac{T V \bar{L}}{\bar{V} \bar{T} L}-\frac{L \bar{T}^{*}}{\bar{L} T^{*}}-\frac{\bar{V} T^{*}}{V \bar{T}}\right)+k_{2}\left(b+d_{L}\right)\left(1-\alpha_{1}\right)\right) \bar{T}^{*} \bar{T}\left(2-\frac{\bar{T}}{T}-\frac{T}{\bar{T}}\right)+k_{2} b \alpha_{1} \bar{T}^{*} \bar{T}\left(3-\frac{\bar{T}}{T}-\frac{T T^{*} \bar{L}}{\bar{T} * \bar{T} L}-\frac{L \bar{T}^{*}}{\bar{L} T^{*}}\right) .
\end{gathered}
$$

The arithmetic-geometric mean inequality $\left(\frac{1}{n} \sum_{i=1}^{n} y_{i} \geq \sqrt[n]{\prod_{i=1}^{n} y_{i}}\right)$ implies $\left.\frac{d Y}{d t}\right|_{(3)} \leq 0$ with equality if and only if $T=\bar{T}, L=\bar{L}, T^{*}=\bar{T}^{*}, V=\bar{V}$. Thus, the largest consistent set in $\left\{\left(T, L, T^{*}, V\right) \in \mathbb{R}_{+}^{4}:\left.\frac{d Y}{d t}\right|_{(3)} \leq 0\right\}$ be the singleton set $\{\bar{E}\}$. It evolves from LaSalle invariance principle that the equilibrium $\bar{E}$ is globally asymptotically stable if $\mathcal{R}_{0}>1$. This completes the proof of the theorem.

\section{Numerical Simulations}

Some mathematical simulations have been executed in this section to explain the stability outcomes and analyze the relative contributions of both viral transmission ways for viral load and latent infected cell reservoir. We considered the proportion of latency fractions is $0.001\left(\alpha_{1}=\alpha_{2}\right)$ while values of all other parameters are given in Table 1 . We computed the basic reproductivity $\mathcal{R}_{0}=0.2<1$ for healthy equilibrium by utilizing our parameters. So it evolves from our outcomes in 
Theorem (3.1) that $E_{0}=\left(10^{6}, 0,0,0\right)$ is globally asymptotically stable. The basic reproductivity for the infected equilibrium is $\mathcal{R}_{0}=2.20>1$ by doing simple arithmetic. Hence, the infected equilibrium $\bar{E}=\left(4.5 \times 10^{5}, 3.90 \times 10^{3}\right.$,
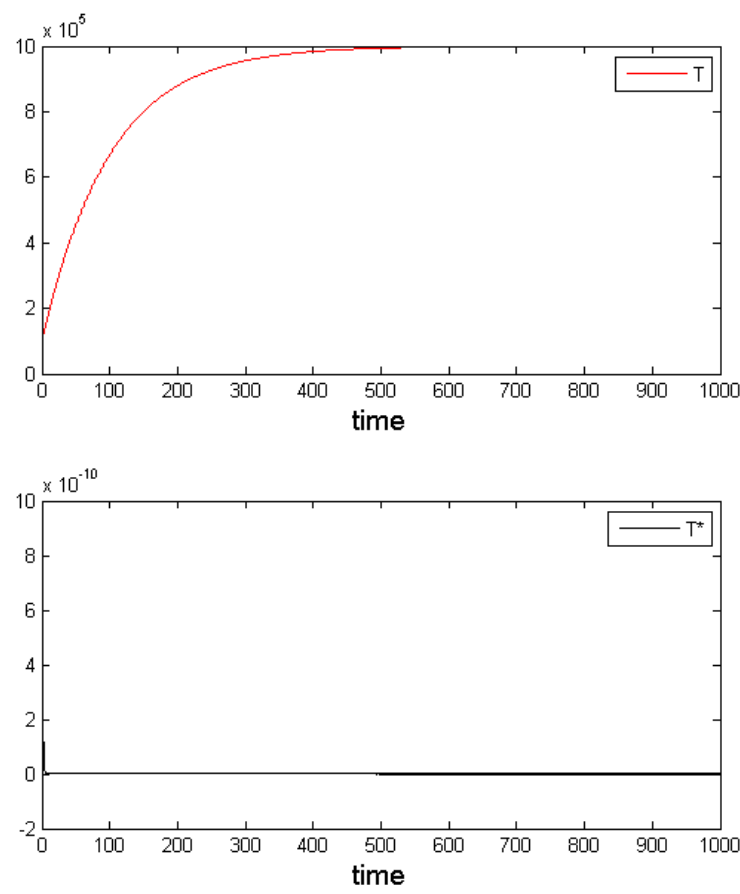

$388.73,3380.28$ ) is globally asymptotically stable by Theorem (3.2). The outcomes of $E_{0}$ and $\bar{E}$ are numerically exhibited in Figure 2 and Figure 3, respectively.
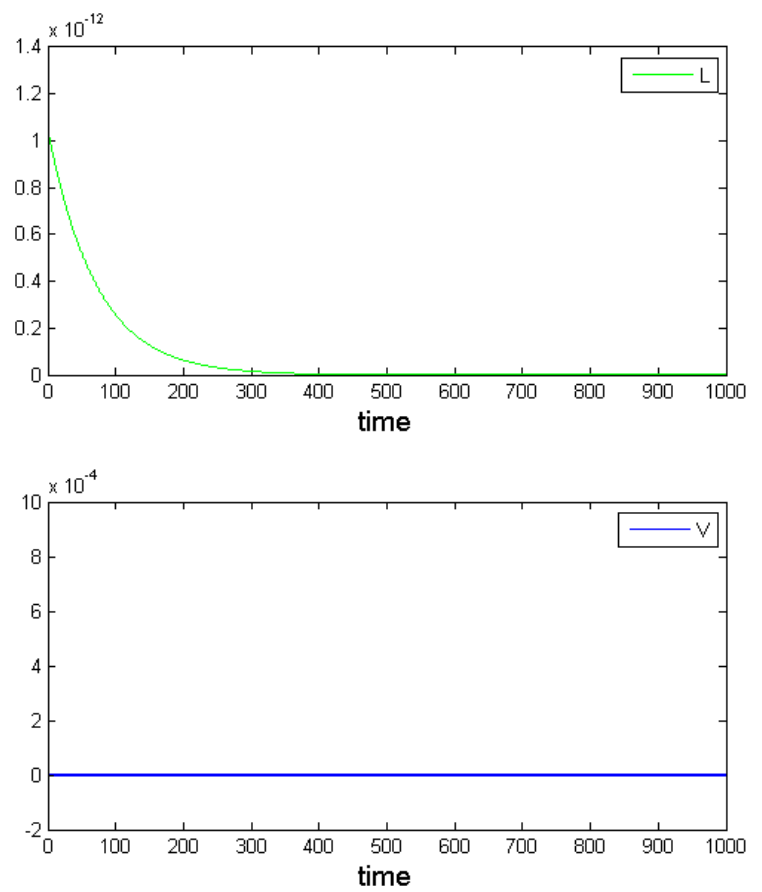

Figure 2. Kinetics anticipated by system (1) for $\mathcal{R}_{0}<1$. We have $\delta=1 ; b=0.01 ; d_{L}=0.004 ; N=100 ; \lambda=10^{4} ; k_{1}=2.4 \times 10^{-10} ; c=23 ; k_{2}=10^{-8}$. IC are $T(0)=10^{5}, L(0)=0, T^{*}(0)=0$ and $V(0)=10^{-3}$.
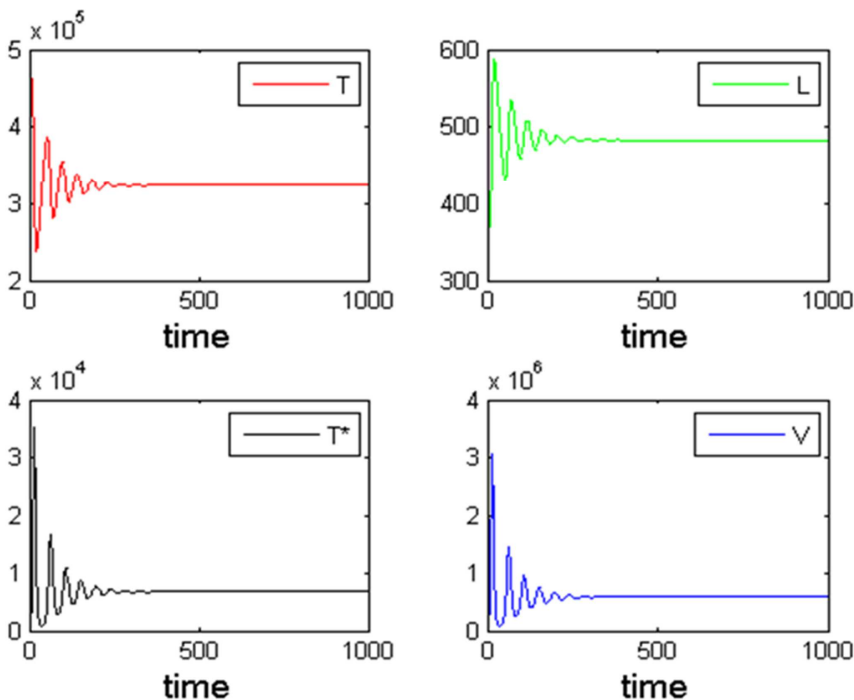

Figure 3. Kinetics anticipated by system (1) for $\mathcal{R}_{0}>1$. We have $\delta=1 ; b=0.01 ; d_{L}=0.004 ; N=2000 ; \lambda=10^{4} ; k_{1}=2.4 \times 10^{-8} ; c=23 ; k_{2}=10^{-6}$. IC are $T(0)=4.4 \times 10^{5}, L(0)=3.88 \times 10^{2}, T^{*}(0)=380$ and $V(0)=3379$.

\section{Relative Contribution (RC)}

The significant proposition is the influence of $\mathrm{CF}$ and $\mathrm{CC}$ on the viral load and the latent containers. We utilized the subsequent fractions $R C_{L}$ and $R C_{V}$ to appraise it.

$$
R C_{L}=\frac{\alpha_{1} k_{1} T V}{\alpha_{2} k_{2} T T^{*}}
$$

and

$$
R C_{V}=\frac{\left(1-\alpha_{1}\right) k_{1} T V}{\left(1-\alpha_{2}\right) k_{2} T T^{*}}
$$

As we know that the proportionality relation between viral load and productively-diseased cells (quasi steady state assumption),

$$
V=\frac{N \delta T^{*}}{c}
$$


then $R C_{L}$ and $R C_{V}$ becomes

$$
R C_{V} \approx \frac{\left(1-\alpha_{1}\right) k_{1} N \delta}{\left(1-\alpha_{2}\right) k_{2} c}
$$

and

$$
R C_{V} \approx \frac{\alpha_{1} k_{1} N \delta}{\alpha_{2} k_{2} c}
$$

Recall that

$$
\begin{aligned}
\mathcal{R}_{0}= & \frac{N k_{1} \lambda}{c d_{T}}\left(\frac{b \alpha_{1}}{b+d_{L}}+\left(1-\alpha_{1}\right)\right) \\
& +\frac{k_{2} \lambda}{\delta d_{T}}\left(\frac{b \alpha_{2}}{b+d_{L}}+\left(1-\alpha_{2}\right)\right)
\end{aligned}
$$

where first and second term represents the basic reproduction number from $\mathrm{CF}$ infection and $\mathrm{CC}$ infection, respectively. As the fractions $\alpha_{1}$ and $\alpha_{2}$ are very small, we had

$$
\frac{\text { basic reproduction number from } \mathrm{CF} \text { infection }}{\text { basic reproduction number from CC infection }} \approx \frac{k_{1} N \delta}{k_{2} c} \text {. }
$$

Therefore, we acquired the following estimates

$$
R C_{V} \approx \frac{\left(1-\alpha_{1}\right)}{\left(1-\alpha_{2}\right)} \cdot \frac{\text { basic reproduction number from CF infection }}{\text { basic reproduction number from CC infection }}
$$

and

$$
R C_{L} \approx \frac{\alpha_{1}}{\alpha_{2}} \cdot \frac{\text { basic reproduction number from CF infection }}{\text { basic reproduction number from CC infection }}
$$

Estimates in equation (14) and (15) agree with the plotted $\mathrm{RC}$ after a very short time. The effect of $\alpha_{1}$ and $\alpha_{2}$ is highly dependent on $R C_{V}$ and $R C_{L}$ from the estimates (14) and (15).
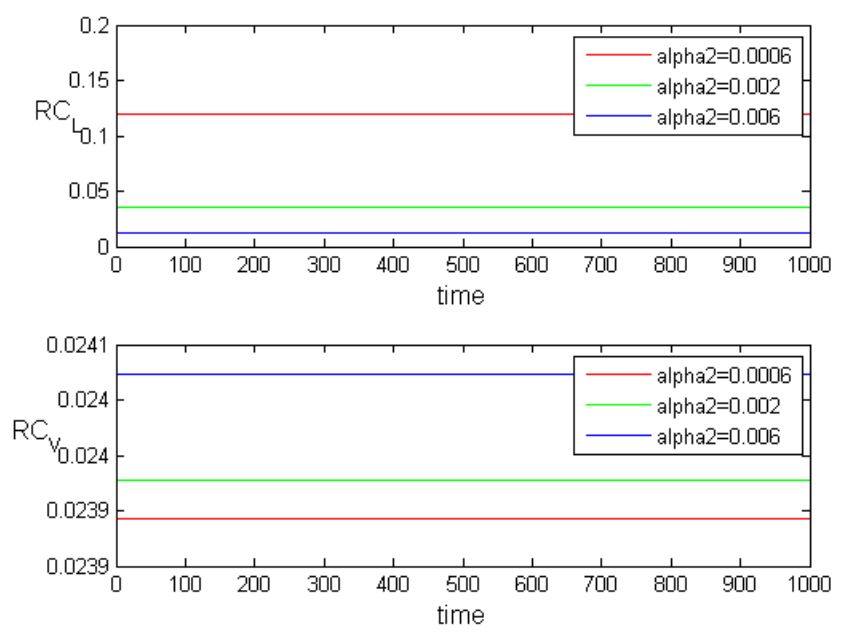

Figure 4. Relative contribution to viral load and infected (latent) cells from $C F$ and CC. Latency fraction $\alpha_{1}$ are $(0.0006 ; 0.002 ; 0.006)$ and $\alpha_{2}=$ 0.003 (fixed): We have $\delta=1 ; b=0.01 ; d_{L}=0.004 ; N=2000 ; \lambda=10^{4}$; $k_{1}=2.4 \times 10^{-8} ; c=23 ; k_{2}=10^{-6}$. IC are $T(0)=10^{5}, L(0)=$ $0, T^{*}(0)=0$ and $V(0)=10^{-3}$.

\section{Conclusion}

HIV studies have determined that virus can infect cells by way of CC and CF [3, 19]. Many HIV mathematical systems have examined virus kinetics with only $C F$ infection. Here, we have globally analyzed an HIV (latency) model containing two types of viral transmission strategies (CF and CC). We have established positivity and boundedness of our system (1) and also obtained the basic reproduction number, $\mathcal{R}_{0}$. We have proved that healthy steady state is globally asymptotically stable if $\mathcal{R}_{0}$ is smaller than 1 and if $\mathcal{R}_{0}$ is larger than 1 , then an unhealthy steady state occurs that is globally asymptotically stable.

We have exhibited the RC of viral transmission strategies (CF and CC) in HIV infection with the assistance of arithmetical and graphical mediums. We have obtained their RC estimates (theoretically) for viral load and latent containers. From which, we considered that viral load and latent containers highly depend on these latency fractions. However, because of uncertain parameter values, RC remains unknown until further investigated.

\section{Future Work}

Currently available HAART therapy is effective in repressing HIV replication beneath the detection limit of conventional clinical examines, but it does not entirely eradicate HIV. Clinical studies show that there is a rapid viral bounce back after the suspension of HAART, which demonstrates the presence of latent reservoirs. To eliminate these viral reservoirs, a shock and kill therapeutic technique has recently been suggested [50]. We may improve our knowledge of the dynamics of the latent reservoir by incorporating the shock and kill approach in our model.

\section{Competing Interests}

The authors declare that there is no conflict of interest.

\section{References}

[1] Agosto, L. M., Zhong, P., Munro, J., and Mothes. Highly active antiretroviral therapies are effective against HIV-1 cellto-cell transmission. PLoS Pathog. 10 (2014), e1003982.

[2] Agosto, L. M., Herring, M. B., Mothes, W., and Henderson, A. J. HIV-1-infected CD4+ T cells facilitate latent infection of resting CD4+ T cells through cell-cell contact. Cell Rep. 24 (2018), 2088-2100.

[3] Sattentau, Q. Avoiding the void: cell-to-cell spread of human viruses. Nat. Rev. Microbiol. 6 (2008), 815-826.

[4] Sigal, A., Kim, J. T., Balazs, A. B., Dekel, E., Mayo, A., Milo, R., and Baltimore, D. Cell-to-cell spread of HIV permits ongoing replication despite antiretroviral therapy. Nature. 477 (2011), 95-98.

[5] Brimacombe, C. L., Grove, J., Meredith, L. W., Hu, K., Syder, A. J., Flores, M. V., Timpe, J. M., Krieger, S. E., Baumert, T. F., Tellinghuisen, T. L., and Wong-Staal F. Neutralizing antibody-resistant hepatitis $\mathrm{C}$ virus cell-to-cell transmission. J. Virol. 85 (2011), 596-605. 
[6] Chen, P., Hubner, W., Spinelli, M. A., and Chen, B. K. Predominant mode of human immunodeficiency virus transfer between $\mathrm{T}$ cells is mediated by sustained Env-dependent neutralization-resistant virological synapses. J. Virol. 81 (2007), 12582-12595.

[7] Sato, H., Orensteint, J., Dimitrov, D., and Martin, M. Cell-tocell spread of HIV-1 occurs within minutes and may not involve the participation of virus particles. Virology. 186 (1992), 712-724.

[8] Johnson, D. C., and Huber, M. T. Directed egress of animal viruses promotes cell-to-cell spread. J. Virol. 76 (2002), 1-8.

[9] Jolly, C., Kashe_, K., Hollinshead, M., and Sattentau, Q. J. HIV-1 cell to cell transfer across an Env-induced, actindependent synapse. J. Exp. Med. 199 (2004), 283-293.

[10] Phillips, D. M. The role of cell-to-cell transmission in HIV infection. AIDS. 8 (1994), 719-732.

[11] Del Portillo, A., Tripodi, J., Najfeld, V., Wodarz, D., Levy, D. N., and Chen, B. K. Multiploid inheritance of HIV-1 during cell-to-cell infection. J. Virol. 85 (2011), 7169-7176.

[12] Iwami, S., Takeuchi, J. S., Nakaoka, S., Mammano, F., Clavel, F., Inaba, H., Kobayashi, T., Misawa, N., Aihara, K., Koyanagi, Y., and Sato, K. Cell-to-cell infection by HIV contributes over half of virus infection. Elife. 4 (2015), e08150.

[13] Komarova, N. L., Anghelina, D., Voznesensky, I., Trinitie, B., Levy, D. N., and Wodarz, D. Relative contribution of freevirus and synaptic transmission to the spread of HIV-1 through target cell populations. Biol. Lett. 9 (2012), DOI: 10.1098/rsbl.2012.1049.

[14] Komarova, N. L., Levy, D. N., and Wodarz, D. Synaptic transmission and the susceptibility of HIV infection to antiviral drugs. Sci. Rep. 3 (2013), DOI: 10.1038/srep02103.

[15] Komarova, N. L., Levy, D. N., and Wodarz, D. Effect of synaptic transmission on viral fitness in HIV infection. PloS one. 7 (2012), e48361.

[16] Komarova, N. L., and Wodarz, D. Virus dynamics in the presence of synaptic transmission. Math. Biosci. 242 (2013), $161-171$.

[17] Pourbashash, H., Pilyugin, S. S., De Leenheer, P., and McCluskey, C. Global analysis of within host virus models with cell-to-cell viral transmission. Discrete Contin. Dyn. Syst. Ser. B 19 (2014), 3341-3357.

[18] Yang, Y., Zou, L., and Ruan, S. Global dynamics of a delayed within-host viral infection model with both virus-to-cell and cell-to-cell transmissions. Math. Biosci. 270 (2015), 183-191.

[19] Guo, T., Qiu, Z., and Rong, L. Analysis of an HIV model with immune responses and cell-to-cell transmission. Bull. Malaysian Math. Sci. Society. 43 (2020), 581-607.

[20] Lai, X., and Zou, X. Modeling HIV-1 virus dynamics with both virus-to-cell infection and cell-to-cell transmission. SIAM J. Appl. Math. 74 (2014), 898-917.

[21] Lai, X., and Zou, X. Modeling cell-to-cell spread of HIV-1 with logistic target cell growth. J. Math. Anal. Appl. 426 (2015), 563-584.

[22] Finzi, D., Hermankova, M., Pierson, T., Carruth, L. M., Buck, C., Chaisson, R. E., Gallant, J., et al. Identification of a reservoir for HIV-1 in patients on highly active antiretroviral therapy. Science. 278 (1997), 1295-1300.

[23] Ruelas, D. S., and Greene, W. C. An integrated overview of HIV-1 latency. Cell. 155 (2013), 519-529.

[24] Strain, M. C., Gunthard, H. F., Havlir, D. V., Ignacio, C. C., Smith, D. M., Leigh-Brown, A. J., Opravil, M., et. al. Heterogeneous clearance rates of long-lived lymphocytes infected with HIV: intrinsic stability predicts lifelong persistence. Proc. Natl. Acad. Sci. 100 (2003), 4819-4824.

[25] Chun, T. W., Carruth, L., Finzi, D., Shen, X., DiGiuseppe, J. A., Taylor, H., Kuo, Y. H., et al. Quantification of latent tissue reservoirs and total body viral load in HIV-1 infection. Nature. 387 (1997), 183-188.

[26] Douek, D. C., Brenchley, J. M., Betts, M. R., Ambrozak, D. R., Hill, B. J., Okamoto, Y., Grossman, Z., et. al. HIV preferentially infects HIV-specific CD4+ T cells. Nature. 417 (2002), 95-98.

[27] Maldarelli, F., Wu, X., Su, L., Simonetti, F. R., Shao, W., Hill, S., Coffin, J. M., et. al. Specific HIV integration sites are linked to clonal expansion and persistence of infected cells. Science. 345 (2014), 179-183.

[28] Alshorman, A., Wang, X., Joseph Meyer, M., and Rong, L. Analysis of HIV models with two time delays. J. Biol. Dyn. 11 (2017), 40-64.

[29] Diekmann, O., Heesterbeek, J. A. P., and Metz, J. A. On the definition and the computation of the basic reproduction ratio $R_{0}$ in models for infectious diseases in heterogeneous populations. J. Math. Biol. 28 (1990), 365-382.

[30] Van den Driessche, P., and Watmough, J. Reproduction numbers and sub-threshold endemic equilibria for compartmental models of disease transmission. Math. Biosci. 180 (2002), 29-48.

[31] Perelson, A. S., Essunger, P., Cao, Y., Vesanen, M., Hurley, A., Saksela, K., Ho, D. D., et. al. Decay characteristics of HIV-1 infected compartments during combination therapy. Nature. 387 (1997), 188-191.

[32] Perelson, A. S., and Nelson, P. W. Mathematical analysis of HIV-1 dynamics in vivo. SIAM Rev. 41 (1999), 3-44.

[33] Perelson, A. S., Neumann, A. U., Markowitz, M., Leonard, J. M., and Ho, D. D. HIV-1 dynamics in vivo: virion clearance rate, infected cell life-span, and viral generation time. Science. 271 (1996), 1582-1586.

[34] James D. Murray. Mathematical Biology. Springer, New York, (1989).

[35] Martin Nowak, Robert May Baron. Virus Dynamics. Oxford University Press, New York, (2000).

[36] Pankavich, S. The effects of latent infection on the dynamics of HIV. Diff. Eq. Dyn. Sys. 24 (2016), 281-303.

[37] Sattentau, Q. J. Cell-to-cell spread of retroviruses. Viruses. 2 (2010), 1306-1321.

[38] Sattentau, Q. J. The direct passage of animal viruses between cells. Curr. Opin. Virol. 1 (2011), 396-402.

[39] Rong, L., Feng, Z., and Perelson, A. S. Mathematical analysis of age-structured HIV-1 dynamics with combination antiretroviral therapy. SIAM J. Appl. Math. 67 (2007), 731756. 
[40] Rong, L., Feng, Z., and Perelson, A. S. Emergence of HIV-1 drug resistance during antiretroviral treatment. Bull. Math. Biol. 69 (2007), 2027-2060.

[41] Rong, L., and Perelson, A. S. Modeling HIV persistence, the latent reservoir, and viral blips. J. Theoret. Biol. 260 (2009), 308-331.

[42] Rong, L., and Perelson, A. S. Modeling latently infected cell activation: viral and latent reservoir persistence, and viral blips in HIV-infected patients on potent therapy. PLoS Comput. Biol. 5 (2009), e1000533.

[43] Rong, L., and Perelson, A. S. Asymmetric division of activated latently infected cells may explain the decay kinetics of the HIV-1 latent reservoir and intermittent viral blips. Math. Biosci. 217 (2009), 77-87.

[44] La Salle, J. P. The stability of dynamical systems. SIAM. (1976).

[45] Zhong, P., Agosto, L. M., Munro, J. B., and Mothes, W. Cell-tocell transmission of viruses. Curr. Opin Virol. 3 (2013), 44-50.
[46] Wen, Q., and Lou, J. The global dynamics of a model about HIV-1 infection in vivo. Ricerche di matematica. 58 (2009), 77-90.

[47] Callaway, S., and Perelson, A. S. HIV-1 infection and low steady state viral loads. Bull. Math. Biol. 64 (2002), 29-64.

[48] Mojaver, A. and Kheiri, H. Mathematical analysis of a class of HIV infection models of CD4+ T-cells with combined antiretroviral therapy. Appl. Math. Comp. 259 (2015), 258270.

[49] Jones, E. and Perelson, A. S. Transient viremia, plasma viral load, and reservoir replenishment in HIV-infected patients on antiretroviral therapy. Def. Synd. 45 (2007), 483-493.

[50] Barouch, D. H., and Deeks, S. G. Immunologic strategies for HIV-1 remission and eradication. Science. 345 (2014), 169174. 